Check for updates

Cite this: RSC Adv., 2017, 7, 53407

Received 11th October 2017

Accepted 14th November 2017

DOI: 10.1039/c7ra11218a

rsc.li/rsc-advances

\section{Unexpected cleavage of thiacalix[4]arene sulfoxides $\uparrow$}

\author{
J. Mikšátko, ${ }^{a}$ V. Eigner ${ }^{b}$ and P. Lhoták (D)*a
}

Thiacalix[4]arenes having one bridge oxidized to a sulfoxide moiety can react with organolithium reagents to form cleaved structures which are otherwise difficult to access. Cleavage of the macrocyclic skeleton is independent of the starting conformation as proven by the same product obtained from the corresponding cone, partial cone or 1,3-alternate derivatives. Quenching of the crude reaction mixture with $\mathrm{D}_{2} \mathrm{O}$ allowed elucidation of the mechanism which is based on the ligand exchange of the sulfoxide functionality. The general applicability of this reaction was demonstrated using the corresponding distal or proximal disulfoxide derivatives, while the quenching of the reaction mixture using various electrophiles allowed the isolation of the corresponding mono- and di-substituted oligomers. The structure of the unexpected product (a tetrameric derivative) was assigned by single crystal $\mathrm{X}$-ray crystallography.

\section{Introduction}

Thiacalix[4]arenes, since their first appearance ${ }^{1}$ in 1997 , have been attracting increasing attention as potential candidates for building blocks and/or molecular scaffolds due to their similarity with the much more well established calix $[n]$ arene family. ${ }^{2}$ The introduction of sulfur atoms in place of the methylene bridges results in novel features or properties ${ }^{3}$ that are impossible (or very difficult) in classical calixarene chemistry. Thus, the stronger directing effects of sulfur compared to the $\mathrm{CH}_{2}$ group offer unusual regioselectivity in the electrophilic aromatic substitution of thiacalix[4]arenes as documented by direct meta nitration ${ }^{4}$ or formylation ${ }^{5}$ (with respect to phenolic oxygens). Moreover, the regio- and stereo-selective $S$-alkylation to sulfonium salts ${ }^{6}$ and chemoselective $S$-oxidation to various sulfoxides or sulfones ${ }^{7}$ represent other unique opportunities inaccessible to the more common calixarene counterpart.

Recently, we studied the partial $S$-oxidation of thiacalix[4] arenes using $\mathrm{NaBO}_{3} \cdot 4 \mathrm{H}_{2} \mathrm{O}$ as the oxidizing agent. ${ }^{8}$ Interestingly, despite the possible formation of many regio- and stereoisomers, the reactions leading to mono- or disulfoxides were highly regio- and stereoselective depending on the starting conformation used. ${ }^{9}$

Synthetic availability of the monosulfoxide derivatives led us to attempt further exploration in the derivatization of thiacalix [4]arenes. The sulfoxide is a versatile moiety which has found

${ }^{a}$ Department of Organic Chemistry, University of Chemistry and Technology, Prague (UCTP), Technicka 5, 16628 Prague 6, Czech Republic. E-mail: lhotakp@vscht.cz; Fax: +420-220444288; Tel: +420-220445055

${ }^{b}$ Solid State Department, UCTP, 16628 Prague 6, Czech Republic

$\dagger$ Electronic supplementary information (ESI) available. CCDC 1554286. For ESI and crystallographic data in CIF or other electronic format see DOI: 10.1039/c7ra11218a
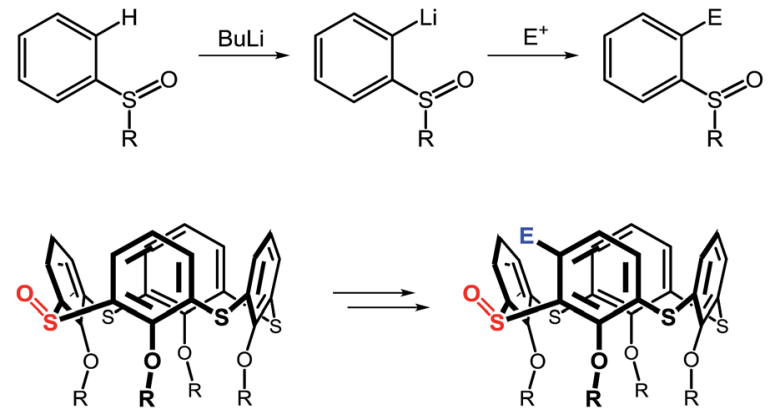

Scheme 1 ortho-Lithiation of sulfoxides and possible application in thiacalix[4]arene chemistry.

numerous applications in organic chemistry, inter alia in orthodirected metallations of aryl sulfoxides. As shown in Scheme 1, the $\mathrm{S}=\mathrm{O}$ functionality is a powerful director in ortho-lithiation ${ }^{10}$ (or ortho-magnesiation ${ }^{11}$ ) which could be used for the introduction of an electrophile moiety into the thiacalixarene skeleton. In this paper we report on our unexpected, yet fortuitous, efforts in the derivatization of the thiacalixarene system.

\section{Results and discussion}

The starting monosulfoxides 1-3 were obtained as recently described $^{9}$ in good yields using the corresponding thiacalixarene conformations and $\mathrm{NaBO}_{3} \cdot 4 \mathrm{H}_{2} \mathrm{O}$ as the oxidizing agent $\left(\mathrm{CHCl}_{3} / \mathrm{AcOH}, 60^{\circ} \mathrm{C}, 4 \mathrm{~h}\right)$. Sulfoxide 1, immobilized in the cone conformation, was subjected to ortho-lithiation reaction (Scheme 2) with $n$-BuLi (10 equiv.) in THF at $-78{ }^{\circ} \mathrm{C}$ and after one hour the corresponding electrophile $\left(\mathrm{I}_{2}\right.$ or DMF) was added. To our surprise, both reactions yielded the same product with no trace of electrophile within the molecule. Instead, we 


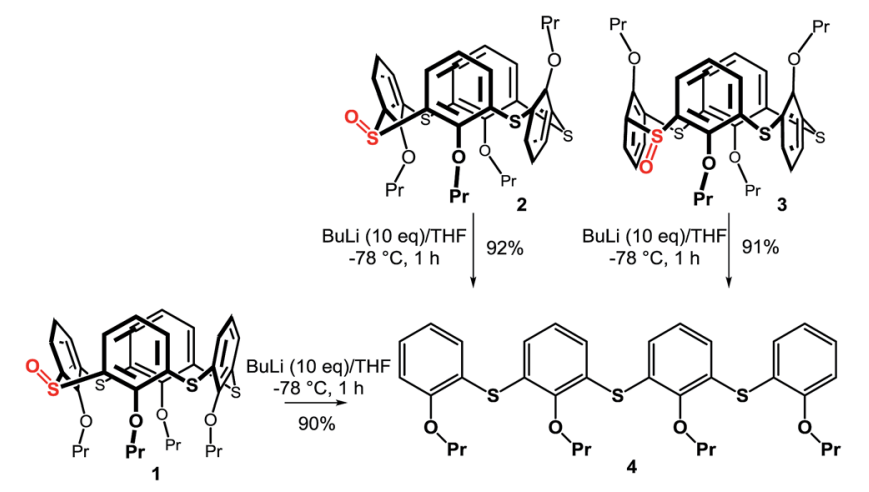

Scheme 2 Attempted ortho-lithiation in several conformations of thiacalix[4]arene.

isolated the linear tetramer 4 (90\% yield) resulting from opening of the thiacalixarene skeleton with the $\mathrm{S}=\mathrm{O}$ functionality being cleaved off. To evaluate the influence of the starting conformation on the unexpected course of the reaction we carried out the same reaction sequence with the partial cone 2 and the 1,3-alternate 3 . In both cases the same product 4 was obtained in high yields ( $92 \%$ and $91 \%$, respectively).

The structure of the products was confirmed by a combination of NMR and MS techniques. The HRMS ESI ${ }^{+}$analysis of 4 showed signals at $m / z=657.21393$ and 673.18726, which were in good agreement with the $[\mathrm{M}+\mathrm{Na}]^{+}(657.21374)$ and $[\mathrm{M}+\mathrm{K}]^{+}$ (673.18768) cations predicted for the cleaved product. The ${ }^{1} \mathrm{H}$ NMR spectrum of $\mathbf{4}\left(\mathrm{CDCl}_{3}\right)$ revealed the presence of seven aromatic signals and two different propyl groups (triplets at 4.11 and $3.93 \mathrm{ppm}$ from $-\mathrm{OCH}_{2}$ - moieties; multiplets at 1.85 and $1.69 \mathrm{ppm}$ from $-\mathbf{C H}_{2} \mathrm{CH}_{3}$; triplets at 1.02 and $0.88 \mathrm{ppm}$ from $-\mathrm{CH}_{2} \mathbf{C H}_{3}$ ) indicating the existence of main symmetry plane in the molecule.

The above results indicate that the cleavage reaction was not dependent on the starting conformation of the thiacalixarene. This enabled us to use monosulfoxide $\mathbf{5}$ for the ensuing study as this derivative is conformationally mobile, and hence, synthetically more easily accessible than sulfoxides 1-3 which have defined conformations. The attempted lithiation of $\mathbf{5}$ under identical reaction conditions ( $n$ - $\mathrm{BuLi}\left(10\right.$ equiv.) in THF at $-78^{\circ} \mathrm{C}$ for $1 \mathrm{~h}$, followed by direct hydrolysis) gave oligophenol ether 6 (see ESI $\dagger$ for the X-ray structure) in $92 \%$ yield (Scheme 3).

As this type of reactivity was unknown in thiacalixarene chemistry we sought to shed light on the mechanism using a range of reaction conditions. Thus, compound $\mathbf{5}$ was reacted with various bases ( 2 equiv.) under standard reaction conditions $\left(\mathrm{THF} /-78{ }^{\circ} \mathrm{C} / 1 \mathrm{~h}\right.$, then quenched with $\left.\mathrm{H}_{2} \mathrm{O}\right)$ to determine what kind of base is the most suitable. The results clearly showed that $n$-BuLi provided the highest yield of 6 ( $43 \%$ yield), while $s$-BuLi and $t$-BuLi gave 6 in 37\% and 5\% yields, respectively. Application of Grignard agents ( $\mathrm{MeMgCl}, \mathrm{PhMgCl}$ ), $\mathrm{NaH}$ or LDA did not invoke any reaction as only starting compound was observed. The use of MeLi gave 6 only in $11 \%$ yield, on the other hand, oligomeric sulfoxide $\mathbf{7 b}$ was isolated as the main product $(49 \%)$ from the reaction mixture. The same type of compound $7 \mathbf{a}$ was also obtained from the crude reaction

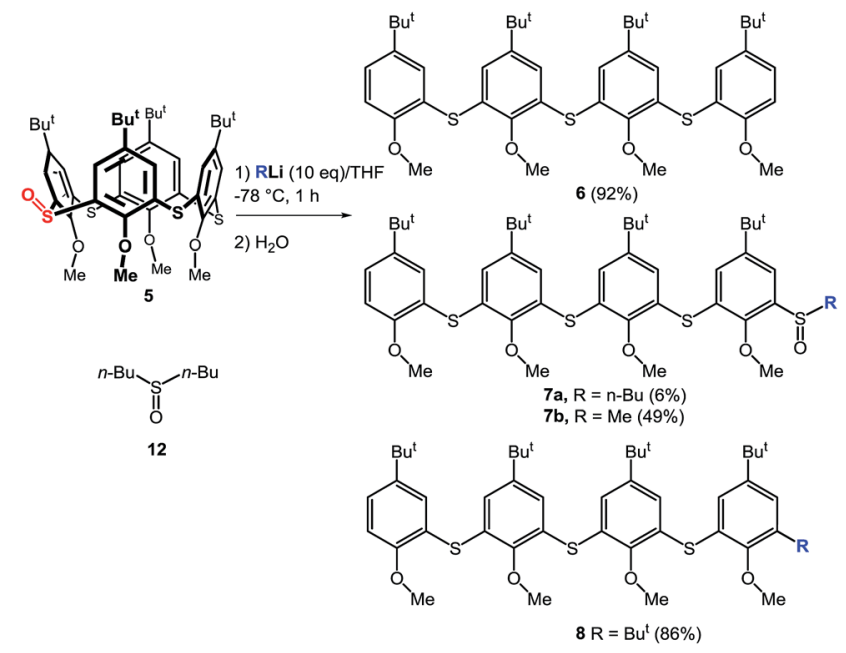

Scheme 3 Cleavage of thiacalix[4]arenes by organolithium compounds.

mixture with $n$-BuLi together with dibutyl sulfoxide 12 (Scheme $3)$. Another type of product was isolated from the reaction with $t$ BuLi, compound $\mathbf{8}$, containing a directly appended tert-butyl group.

The structure of $\mathbf{7 b}$ was supported by the presence of four singlets from the tert-butyl groups in the ${ }^{1} \mathrm{H}$ NMR spectrum $\left(\mathrm{CDCl}_{3}\right)$ at $1.27,1.24,1.08$, and $1.07 \mathrm{ppm}$, together with four singlets from $\mathrm{OCH}_{3}-$ moieties (3.93, 3.92, 3.91, and $3.83 \mathrm{ppm}$ ), confirming the absence of any symmetry elements. The methyl group of the sulfoxide moiety could be found as a singlet at $2.80 \mathrm{ppm}$. The HRMS ESI ${ }^{+}$analysis of $7 \mathbf{b}$ revealed signals at $\mathrm{m} / \mathrm{z}$ $=809.33952,831.32160$, and 847.29315, which were in excellent agreement with the predicted values for the $[\mathrm{M}+\mathrm{H}]^{+}$ (809.33963), $[\mathrm{M}+\mathrm{Na}]^{+}(831.32158)$, and $[\mathrm{M}+\mathrm{K}]^{+}(847.29552)$ cations.

The above described results indicate that cleavage of the thiacalixarene derivatives could proceed via a ligand exchange mechanism (Scheme 4) which is documented for the reaction of sulfoxides with organolithium and Grignard reagents. ${ }^{12}$ These substitution reactions are considered to proceed via an $\mathrm{S}_{\mathrm{N}} 2$-type process involving the formation of a hypervalent sulfurane, ${ }^{13}$ as a transition state complex rather than an intermediate (structure B in the tentative mechanism shown in Scheme 5). Substitution of aryl moiety with alkyls ( $\mathrm{Me}, \mathrm{Bu}$ ) is plausible because of strain release due to the macrocyclic arrangement

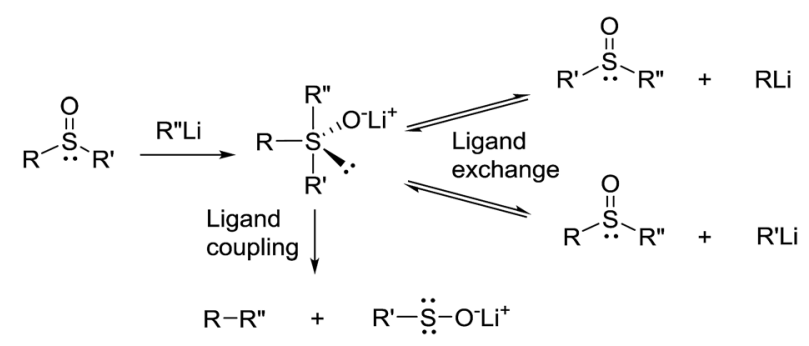

Scheme 4 Reactions of sulfoxides with organolithium agents. 


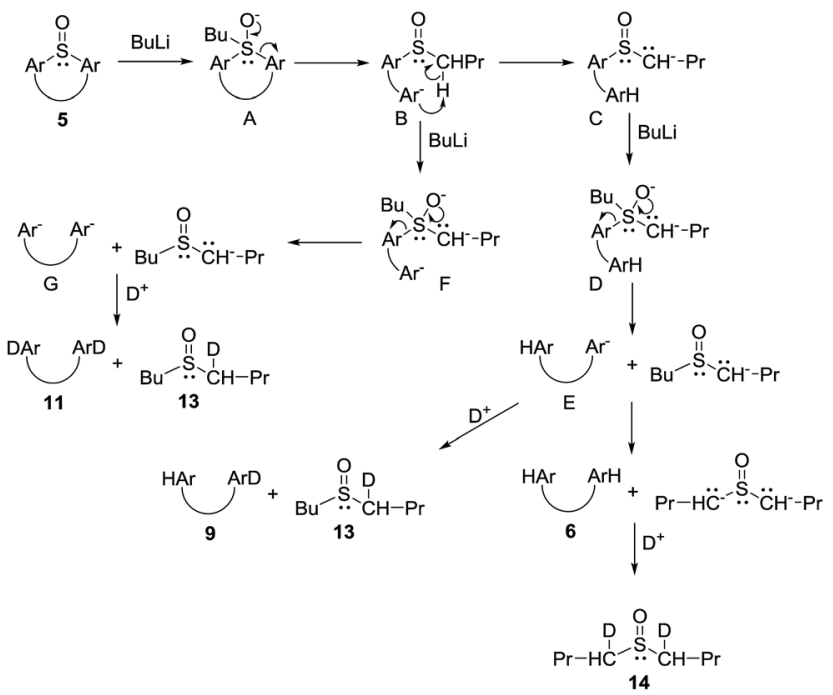

Scheme 5 Tentative mechanism for the cleavage reaction of thiacalix [4]arenes by $n$-BuLi.

around the sulfoxide moiety-thus leading directly to cleaved structure $\mathrm{C}$ corresponding to products $7 \mathbf{a}, \mathbf{7 b}$. On the other hand, the formation of derivative 8 suggested a ligand coupling procedure (Scheme 4), which has been reported for some sterically demanding sulfoxides. ${ }^{14}$

To further study the mechanism, we carried out a series of experiments where the reaction mixture was quenched with $\mathrm{D}_{2} \mathrm{O}$. Interestingly, the reaction products quenched after stirring for $1 \mathrm{~h}$ contained no deuterium on the aromatic moieties instead providing $90 \%$ yield of 6 . However, HRMS analysis revealed the presence of dideuterated dibutyl sulfoxide $\mathbf{1 4}$ (Scheme 6). ${ }^{15}$ This indicated that the aromatic anions (B and E), formed by the cleavage of the hypervalent sulfuran species (A and $\mathrm{D}$ ), were quenched by the acidic hydrogens ( $\alpha$-positions of butyl sulfoxide byproduct) before the addition of $\mathrm{D}_{2} \mathrm{O}$ thus providing only deuterated sulfoxide (Scheme 5).

The above reaction is very fast as indicated by quenching $\left(\mathrm{D}_{2} \mathrm{O}\right)$ the reaction mixture after only a 2 min treatment with $n$ BuLi. Under these conditions a mixture of monodeuterated and

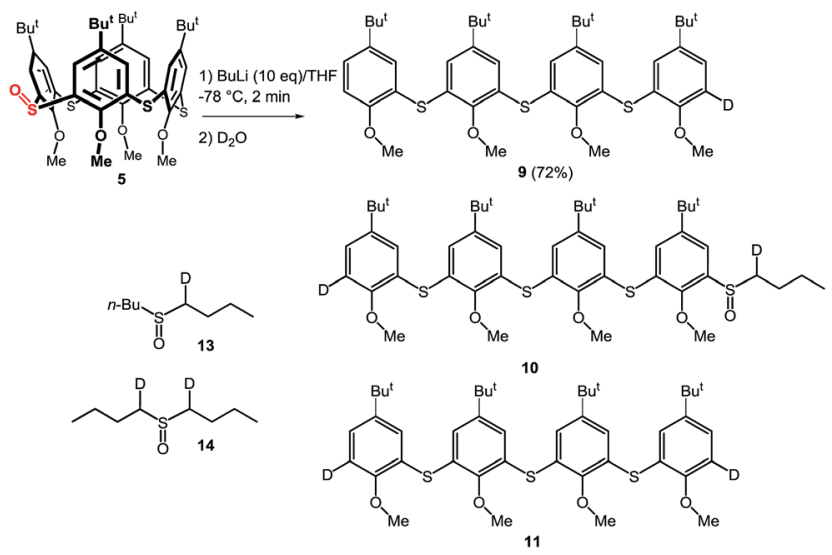

Scheme 6 Cleavage of thiacalix[4]arenes-deuteration experiments. dideuterated tetramers $\mathbf{9}$ and $\mathbf{1 1}$ was obtained in $72 \%$ yield together with small amount of 10, accompanied by mono- and di-deuterated dibutyl sulfoxide derivatives $\mathbf{1 3}$ and $\mathbf{1 4}$ (Scheme 6). If the same reaction was quenched after 30 seconds very similar results were obtained. This indicated that the formation of aromatic dianion $\mathrm{G}$ or the corresponding hypervalent sulfuran species $\mathrm{F}$ is extremely fast and both species gradually disappear from the reaction mixture (Scheme 5).

To show the general applicability of this type of cleavage the corresponding thiacalix[4]arene sulfoxides $\mathbf{1 5}$ and 16 (Scheme 7) were subjected to similar reaction conditions ( $n$-BuLi (10 equiv.) in THF at $-78{ }^{\circ} \mathrm{C}$ for $30 \mathrm{~min}$, followed by direct hydrolysis). As expected, the proximal regioisomer 15 smoothly furnished the appropriate trimer $\mathbf{1 7}$ that was isolated in $89 \%$ yield after preparative TLC. The concomitant formation of the corresponding p-tert butyl anisol (not isolated) was also observed. Similarly, the distal disulfoxide $\mathbf{1 6}$ was cleaved to dimer 18 in $90 \%$ isolated yield. Both results strongly suggest that the $n$-BuLi-induced cleavage of the thiacalix[4]arene skeleton is not limited to only monosulfoxide species, but, in fact, can be successfully applied to a more highly oxidized skeletons.

To further show the usefulness of this approach we have carried out a series of experiments where the crude reaction mixture (after the addition of $n$-BuLi) was quenched by various electrophiles instead of water. Our previous experiments confirmed that the formation of monoanion $\mathrm{E}$ or dianion $\mathrm{G}$ (Scheme 5) is very fast, while the longer reaction times with BuLi led to their complete disappearance due to the quenching by acidic $\alpha$-protons of dibutyl sulfoxide formed as a byproduct. Thus, the stirring of starting monosulfoxide 5 with $n$-BuLi for 20 min led only to the formation of tetramer 6 without any products formed by the reaction with electrophiles added. This indicated that both monoanion $\mathrm{E}$ and dianion $\mathrm{G}$ are no longer present in the reaction mixture at the time of electrophile addition. Based on this finding we set the reaction time with $n$-BuLi to only 30 seconds to secure the presence of $\mathrm{E}$ and $\mathrm{G}$, than the corresponding electrophile (benzaldehyde, benzophenone, ethyl chloroformate, $\mathrm{CO}_{2}$ ), was added and the reaction mixture was stirred 10 minutes at $-78^{\circ} \mathrm{C}$, the cooling bath was removed, and the reaction mixture was allowed to achieve the
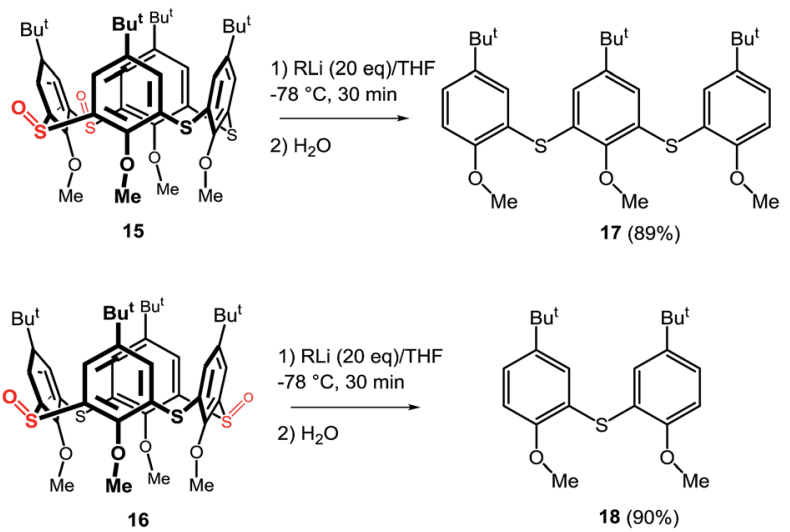

Scheme 7 Cleavage of thiacalix[4]arene disulfoxides. 

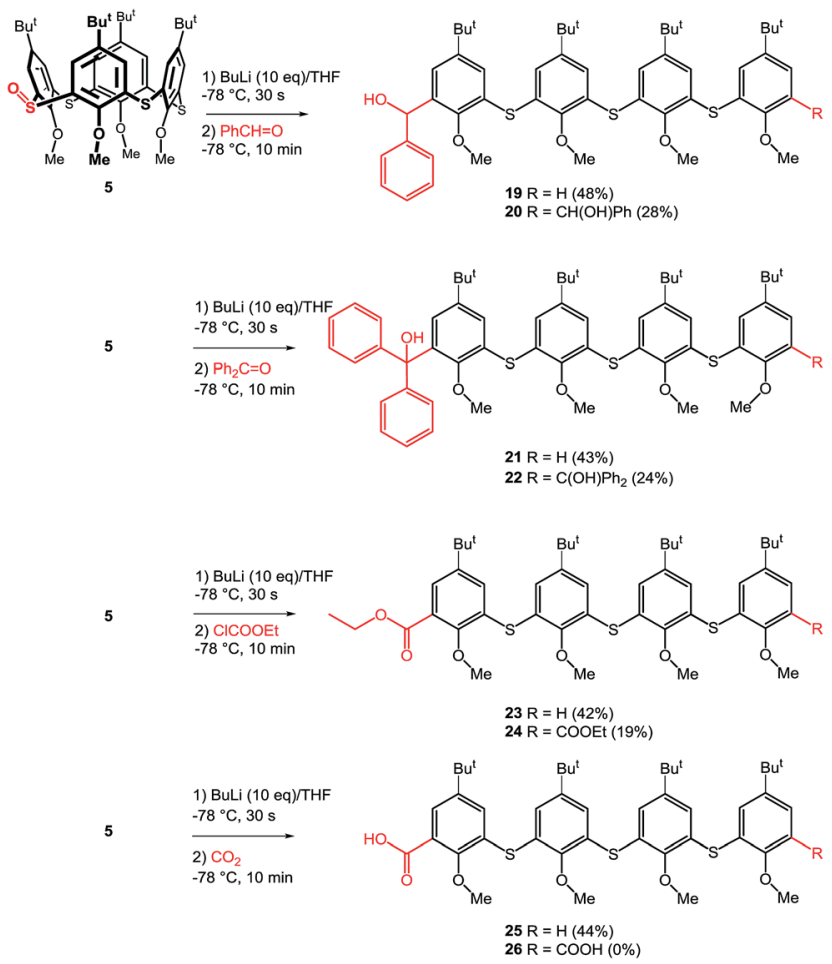

Scheme 8 Quenching with various electrophiles.

room temperature. Using this procedure (Scheme 8) the formation of the corresponding monosubstituted oligomers 19, 21,23 , and 25 was observed in $48 \%, 43 \%, 42 \%$, and $44 \%$ yield, respectively. In all the cases, the main products (mono-) were accompanied by the corresponding disubstituted oligomers 20, 22, 24 and 26 in approximately $2: 1$ ratio. The only exception is represented by the diacid 26 which was obtained in only very minor amount and was not isolated in enough pure form to allow full characterization.

All the above prepared oligomers are so far unknown compounds which are difficult to access. The tert-butylated compounds $\mathbf{6}$ and $\mathbf{1 7}$ could be potentially obtainable using the step-by-step condensation of the $p$-tert-butylphenol with $\mathrm{SCl}_{2}$ to form the corresponding tetraphenolic or triphenolic precursors as described by Sone et al. ${ }^{16}$ On the other hand, the same procedure would be inapplicable for the synthesis of tetramer 4 due to the nonselective reaction of starting phenol in position 2(6) or 4. From this point of view, the cleavage of thiacalixarene sulfoxides offers relatively straightforward and general way for this kind of products, providing the final tetramers in very high yields. Potential utilization of the above tetrameric derivatives can be envisaged in their application as building blocks in the design and synthesis of bigger thiacalixarene-based structures.

\section{Conclusions}

In conclusion, thiacalix[4]arenes having one bridge oxidized to the sulfoxide can react with organolithium compounds (best results obtained with $n$-BuLi) to form cleaved linear structures which are otherwise highly difficult to access. The structure of one such an unexpected product (tetrameric derivative) was assigned by single crystal structure determination. Cleavage of the macrocyclic skeleton is independent of the starting conformation as shown by the same product obtained from the corresponding cone, partial cone or 1,3-alternate derivatives. The quenching of the crude reaction mixture with $\mathrm{D}_{2} \mathrm{O}$ allowed us to propose a plausible mechanism which is based on ligand exchange on the sulfoxide moiety. The general applicability of this reaction was demonstrated using the corresponding distal or proximal disulfoxide derivatives. Quenching the reaction mixture using various electrophiles allowed for the isolation of the corresponding mono- and di-substituted oligomers. Further synthetic applications of this unusual behavior are currently under study and will be reported in due course.

\section{Experimental}

\section{General experimental procedures}

All chemicals were purchased from commercial sources and used without further purification. Solvents were dried and distilled using conventional methods. All samples were dried in the desiccator over $\mathrm{P}_{2} \mathrm{O}_{5}$ under vacuum (1 Torr) at $80{ }^{\circ} \mathrm{C}$ for 8 hours. NMR spectra were performed on Agilent 400-MR DDR2 $\left({ }^{1} \mathrm{H}: 400 \mathrm{MHz},{ }^{13} \mathrm{C}: 100 \mathrm{MHz}\right)$ spectrometer. Chemical shifts $(\delta)$ are expressed in ppm and are referred to the residual peak of the solvent or TMS as an internal standard; coupling constants $(J)$ are in $\mathrm{Hz} . \mathrm{d}^{1}$-Chloroform (stabilized over silver wool, $99.8 \%$ of deuterium) was used for the spectroscopic studies. The signal assignment was supported by ${ }^{1} \mathrm{H}^{-}{ }^{1} \mathrm{H}$ COSY, ${ }^{1} \mathrm{H}^{-13} \mathrm{C}$ HMQC or ${ }^{1} \mathrm{H}^{-13} \mathrm{C}$ HMBC $2 \mathrm{D}$ NMR using the standard pulse sequences provided by Bruker. The mass analyses were performed using ESI technique on Q-TOF (Micromass) spectrometer. Melting points were measured on Heiztisch Mikroskop - Polytherm A (Wagner \& Munz, Germany) and were not corrected. The IR spectra were measured on an FT-IR spectrometer Nicolet 740 or Bruker IFS66 spectrometers equipped with a heatable Golden Gate Diamante ATR-Unit (SPECAC) in KBr. 100 scans for one spectrum were co-added at a spectral resolution of $4 \mathrm{~cm}^{-1}$. The courses of the reactions were monitored by TLC using TLC aluminium sheets with silica gel 60 F254 (Merck). Thin layer chromatography (TLC) was performed using glass sheets $(25 \times$ $25 \mathrm{~cm}$ ) coated with silica gel $60 \mathrm{~F} 254$, followed by visualization with UV light.

\section{Synthetic procedures}

General procedure for cleavage of thiacalixarene derivatives. Thiacalixarene monosulfoxide $(0.063 \mathrm{mmol})$ was added to a $25 \mathrm{~mL}$ Schlenk flask and put under high vacuum. After 20 minutes, the flask was filled several times with argon and anhydrous THF $(5 \mathrm{~mL})$ was added through septum. The solution was cooled down to $-78{ }^{\circ} \mathrm{C}$ and $n$-butyllithium (10 eq., $2 \mathrm{M}$ in THF) was added dropwise to the flask. The solution was stirred for $1 \mathrm{~h}$ at $-78{ }^{\circ} \mathrm{C}$, then removed from cooling bath and the reaction was quenched by addition of distilled water $(3 \mathrm{~mL})$. The mixture was extracted with dichloromethane $(3 \times 15 \mathrm{~mL})$ and brine. Separated organic layers were washed with water $(50 \mathrm{~mL})$ 
and dried over $\mathrm{MgSO}_{4}$. The solvent was removed under reduced pressure to yield crude product which was further purified using thin layer chromatography with dichloromethane as an eluent.

Compound 4. The general procedure was applied to compound 1 ( 2 or 3 ). The product 4 was isolated after preparative TLC as a colourless oil in $90 \%$ yield ( $42 \mathrm{mg}$ ), ( $92 \%$ from 2 , 91\% from 3). ${ }^{1} \mathrm{H}$ NMR (400 MHz, $\left.\mathrm{CDCl}_{3}, 296 \mathrm{~K}\right) \delta$ (ppm): 7.32 $(2 \mathrm{H}, \mathrm{dd}, J=7.5,1.4 \mathrm{~Hz}, \mathrm{Ar}-\mathbf{H}), 7.29(2 \mathrm{H}, \mathrm{td}, J=7.7,1.6 \mathrm{~Hz}, \mathrm{Ar}-\mathbf{H})$, $6.91(6 \mathrm{H}, \mathrm{m}, \mathrm{Ar}-\mathrm{H}), 6.84(2 \mathrm{H}, \mathrm{t}, J=7.7 \mathrm{~Hz}, \mathrm{Ar}-\mathrm{H}), 6.78(2 \mathrm{H}, \mathrm{dd}, J=$ 7.7, 1.6 Hz, Ar-H), $4.11\left(4 \mathrm{H}, \mathrm{t}, J=6.7 \mathrm{~Hz},-\mathbf{O C H}_{2}-\right), 3.93(4 \mathrm{H}, \mathrm{t}, J$ $\left.=6.4 \mathrm{~Hz},-\mathrm{OCH}_{2}^{-}\right), 1.85\left(4 \mathrm{H}, \mathrm{m},-\mathbf{C H}_{2} \mathrm{CH}_{3}\right), 1.69(4 \mathrm{H}, \mathrm{m}$, $\left.-\mathbf{C H}_{2} \mathrm{CH}_{3}\right), 1.02\left(6 \mathrm{H}, \mathrm{t}, J=7.5 \mathrm{~Hz},-\mathrm{CH}_{2} \mathbf{C H}_{3}\right), 0.88(6 \mathrm{H}, \mathrm{t}, J=$ $\left.7.5 \mathrm{~Hz},-\mathrm{CH}_{2} \mathbf{C H}_{3}\right) ;{ }^{13} \mathrm{C}$ NMR (100 MHz, $\left.\mathrm{CDCl}_{3}, 296 \mathrm{~K}\right) \delta(\mathrm{ppm})$ : 158.3 , 155.5, 134.4, 131.6, 130.0, 129.6, 129.5, 129.0, 124.9, 121.8, 121.2, 112.2, 74.8, 70.2, 23.7, 23.6, $10.6(2 \times)$; IR $(\mathrm{KBr}) \nu$ 2961, 2924, 2874, 2850, 1577, 1464, 1426, 1382, 1273, 1240, 1224, 1067, 977, $747 \mathrm{~cm}^{-1}$. HRMS (ESI $\left.{ }^{+}\right):\left(\mathrm{C}_{36} \mathrm{H}_{42} \mathrm{O}_{4} \mathrm{~S}_{3}\right) \mathrm{m} / z$ calcd: 657.21374 [M+ Na $]^{+}, 673.18768[\mathrm{M}+\mathrm{K}]^{+}$; found: $657.21393[\mathrm{M}+$ $\mathrm{Na}]^{+}, 673.18726[\mathrm{M}+\mathrm{K}]^{+}$.

Compounds 6 and 7a. The general procedure was applied to compound 5. The product 6 (43 $\mathrm{mg}, 92 \%)$ was isolated after preparative TLC as a white foam, mp: $61-63{ }^{\circ} \mathrm{C}$, together with small amount of oligomeric sulfoxide $7 \mathbf{a}(3 \mathrm{mg}, 6 \%)$ in the form of colourless oil.

Data for 6. ${ }^{1} \mathrm{H}$ NMR (400 MHz, $\left.\mathrm{CDCl}_{3}, 296 \mathrm{~K}\right) \delta(\mathrm{ppm}): 7.29$ $(2 \mathrm{H}, \mathrm{dd}, J=8.61,2.35 \mathrm{~Hz}, \mathrm{Ar}-\mathrm{H}), 7.26(2 \mathrm{H}, \mathrm{d}, J=2.35 \mathrm{~Hz}, \mathrm{Ar}-\mathbf{H})$, $6.98(2 \mathrm{H}, \mathrm{d}, J=2.35 \mathrm{~Hz}, \operatorname{Ar}-\mathbf{H}), 6.88(2 \mathrm{H}, \mathrm{d}, J=2.35 \mathrm{~Hz}, \operatorname{Ar}-\mathbf{H})$, $6.87(2 \mathrm{H}, \mathrm{d}, J=8.22 \mathrm{~Hz}, \mathrm{Ar}-\mathbf{H}), 3.93\left(6 \mathrm{H}, \mathrm{s},-\mathrm{OCH}_{3}\right), 3.83(6 \mathrm{H}, \mathrm{s}$, $\left.-\mathrm{OCH}_{3}\right), 1.23$ (18H, s, $\boldsymbol{t}$-Bu), 1.07 (18H, s, t-Bu); ${ }^{13} \mathrm{C}$ NMR (100 $\left.\mathrm{MHz}, \mathrm{CDCl}_{3}, 296 \mathrm{~K}\right) \delta$ (ppm): 156.2, 154.5, 147.7, 144.1, 130.7, 129.8, 128.7, 127.7, 127.1, 125.8, 121.4, 110.6, 60.8, 55.9, 34.5, 34.1, 31.4, 31.1; IR (KBr) $\nu$ 2958, 2904, 2867, 1549, 1489, 1458, 1416, 1392, 1260, 1069, 999, 872, $807 \mathrm{~cm}^{-1}$. HRMS $\left(\mathrm{ESI}^{+}\right)$: $\left(\mathrm{C}_{44} \mathrm{H}_{58} \mathrm{O}_{4} \mathrm{~S}_{3}\right) \mathrm{m} / z$ calcd: $769.33864[\mathrm{M}+\mathrm{Na}]^{+}, 785.31288[\mathrm{M}+\mathrm{K}]^{+}$, found: $769.33960[\mathrm{M}+\mathrm{Na}]^{+}, 785.31231[\mathrm{M}+\mathrm{K}]^{+}$.

Data for $7 \boldsymbol{a} .{ }^{1} \mathrm{H}$ NMR (400 MHz, $\left.\mathrm{CDCl}_{3}, 296 \mathrm{~K}\right) \delta(\mathrm{ppm}): 7.75$ $(1 \mathrm{H}, \mathrm{d}, J=2.4 \mathrm{~Hz}, \mathrm{Ar}-\mathrm{H}), 7.32(1 \mathrm{H}, \mathrm{d}, J=2.4 \mathrm{~Hz}, \operatorname{Ar}-\mathbf{H}), 7.31(1 \mathrm{H}$, $\mathrm{dd}, J=8.5,2.4 \mathrm{~Hz}, \mathrm{Ar}-\mathrm{H}), 7.28(1 \mathrm{H}, \mathrm{d}, J=2.4 \mathrm{~Hz}, \operatorname{Ar}-\mathbf{H}), 7.00(1 \mathrm{H}$, $\mathrm{d}, J=2.4 \mathrm{~Hz}, \mathrm{Ar}-\mathbf{H}), 6.97(1 \mathrm{H}, \mathrm{d}, J=2.4 \mathrm{~Hz}, \operatorname{Ar}-\mathbf{H}), 6.89(1 \mathrm{H}, \mathrm{d}, J=$ $2.4 \mathrm{~Hz}, \operatorname{Ar}-\mathbf{H}), 6.88(1 \mathrm{H}, \mathrm{d}, J=8.5 \mathrm{~Hz}, \operatorname{Ar}-\mathbf{H}), 6.81(1 \mathrm{H}, \mathrm{d}, J=$ $2.4 \mathrm{~Hz}, \mathrm{Ar}-\mathrm{H}), 3.93\left(3 \mathrm{H}, \mathrm{s},-\mathrm{OCH}_{3}\right), 3.92\left(3 \mathrm{H}, \mathrm{s},-\mathrm{OCH}_{3}\right), 3.91$ $\left(3 \mathrm{H}, \mathrm{s},-\mathbf{O C H}_{3}\right), 3.83\left(3 \mathrm{H}, \mathrm{s},-\mathbf{O C H}_{3}\right), 3.05\left(1 \mathrm{H}, \mathrm{m}, \mathrm{O}-\mathbf{C H}_{2}-\right), 2.76$ $\left(1 \mathrm{H}, \mathrm{m}, \mathrm{O}-\mathbf{C H}_{2}-\right), 1.85\left(1 \mathrm{H}, \mathrm{m},-\mathbf{C H}_{2}{ }^{-}\right), 1.63\left(1 \mathrm{H}, \mathrm{m},-\mathbf{C H}_{2}{ }^{-}\right), 1.44$ $\left(2 \mathrm{H}, \mathrm{m},-\mathbf{C H}_{2} \mathrm{CH}_{3}\right), 1.27(9 \mathrm{H}, \mathrm{s}, \boldsymbol{t}$-Bu) $1.24(9 \mathrm{H}, \mathrm{s}, \boldsymbol{t}-\mathbf{B u}), 1.08$ (9H, s, t-Bu), 1.07 (9H, s, $\boldsymbol{t}$-Bu), $0.93\left(3 \mathrm{H}, \mathrm{t}, J=7.4 \mathrm{~Hz},-\mathrm{CH}_{2} \mathbf{C H}_{3}\right.$ ); ${ }^{13} \mathrm{C} \mathrm{NMR}\left(100 \mathrm{MHz}, \mathrm{CDCl}_{3}, 296 \mathrm{~K}\right) \delta(\mathrm{ppm}): 156.5,154.9,154.3$, $152.5,149.4,148.3,148.0,144.3,137.5,133.3,131.1,130.5$, $129.9,129.0,128.2,127.9,127.9,127.7,127.5,126.7,126.2$, 121.9, 121.1, 110.8, 61.8, 61.0, 61.0, 56.1, 55.1, 35.2, 34.7, 34.6, 34.3, 31.5, 31.3, 31.2, 31.2, 24.5, 22.0, 13.8; IR (KBr) $\nu$ 2960, 2888, 1459, 1417, 1262, 1244, 1066, 996, 907, 729, $645 \mathrm{~cm}^{-1}$. HRMS $\left(\mathrm{ESI}^{+}\right):\left(\mathrm{C}_{48} \mathrm{H}_{66} \mathrm{O}_{5} \mathrm{~S}_{4}\right) \mathrm{m} / z$ calcd: $873.36853[\mathrm{M}+\mathrm{Na}]^{+}, 889.34247$ $[\mathrm{M}+\mathrm{K}]^{+}$, found: $873.36877[\mathrm{M}+\mathrm{Na}]^{+}, 889.34261[\mathrm{M}+\mathrm{K}]^{+}$.

Compound $7 \mathbf{b}$. The general procedure was applied to compound 5 using $\mathrm{MeLi}\left(1.6 \mathrm{M}\right.$ in $\mathrm{Et}_{2} \mathrm{O}$ ) instead of BuLi. The sulfoxide $\mathbf{7 b}$ was isolated from the reaction mixture as the main product (49\%, colourless oil). ${ }^{1} \mathrm{H} \mathrm{NMR}\left(400 \mathrm{MHz}, \mathrm{CDCl}_{3}, 296 \mathrm{~K}\right)$ $\delta(\mathrm{ppm}): 7.78(1 \mathrm{H}, \mathrm{d}, J=2.4 \mathrm{~Hz}, \operatorname{Ar}-\mathbf{H}), 7.31$ (2H, m, Ar-H), 7.29 $(1 \mathrm{H}, \mathrm{d}, J=2.4 \mathrm{~Hz}, \mathrm{Ar}-\mathrm{H}), 7.00(1 \mathrm{H}, \mathrm{d}, J=2.4 \mathrm{~Hz}, \mathrm{Ar}-\mathrm{H}), 6.98(1 \mathrm{H}$, $\mathrm{d}, J=2.4 \mathrm{~Hz}, \mathrm{Ar}-\mathrm{H}), 6.89(1 \mathrm{H}, \mathrm{d}, J=2.4 \mathrm{~Hz}, \mathrm{Ar}-\mathbf{H}), 6.88(1 \mathrm{H}, \mathrm{d}, J=$ $8.6 \mathrm{~Hz}, \mathrm{Ar}-\mathbf{H}), 6.83(1 \mathrm{H}, \mathrm{d}, J=2.4 \mathrm{~Hz}, \mathrm{Ar}-\mathbf{H}), 3.93\left(3 \mathrm{H}, \mathrm{s},-\mathbf{O C H}_{3}\right)$, $3.92\left(3 \mathrm{H}, \mathrm{s},-\mathrm{OCH}_{3}\right), 3.91\left(3 \mathrm{H}, \mathrm{s},-\mathrm{OCH}_{3}\right), 3.83\left(3 \mathrm{H}, \mathrm{s},-\mathrm{OCH}_{3}\right)$, $2.80\left(3 \mathrm{H}, \mathrm{s},-\mathrm{SO}-\mathbf{C H}_{3}\right), 1.27$ (9H, s, $\boldsymbol{t}$-Bu), 1.24 (9H, s, $\boldsymbol{t}$-Bu), 1.08 (9H, s, $\boldsymbol{t}$-Bu), 1.07 (9H, s, $\boldsymbol{t}$-Bu); ${ }^{13} \mathrm{C} \mathrm{NMR}\left(100 \mathrm{MHz}, \mathrm{CDCl}_{3}, 296\right.$ K) $\delta(\mathrm{ppm}): 156.5,154.9,154.5,152.2,149.6,148.3,148.0,144.3$, 139.1 , 133.2, 131.2, 130.5, 130.0, 128.7, 128.2, 128.0, 128.0, $127.9,127.5,127.0,126.2,121.1,121.0,110.8,61.8,61.0(2 \times)$, $56.1,42.4,35.3,34.7,34.6,34.3,31.5,31.4,31.2,31.2$; IR (KBr) $\nu$ 2960, 2871, 1547, 1459, 1416, 1262, 1244, 1066, 996, 907, 729, $643 \mathrm{~cm}^{-1}$. HRMS $\left(\mathrm{ESI}^{+}\right):\left(\mathrm{C}_{45} \mathrm{H}_{60} \mathrm{O}_{5} \mathrm{~S}_{4}\right) \mathrm{m} / z$ calcd: $809.33963[\mathrm{M}+$ $\mathrm{H}]^{+}, 831.32158[\mathrm{M}+\mathrm{Na}]^{+}, 847.29552[\mathrm{M}+\mathrm{K}]^{+}$, found: 809.33952 $[\mathrm{M}+\mathrm{H}]^{+}, 831.32160[\mathrm{M}+\mathrm{Na}]^{+}, 847.29315[\mathrm{M}+\mathrm{K}]^{+}$.

Compound 8. The general procedure was applied to compound 5 using $t$-BuLi (1.9 M in pentane) instead of $n$-BuLi. The $t$-butylated tetramer $\mathbf{8}$ was isolated as the main product (66\%, colourless oil). ${ }^{1} \mathrm{H}$ NMR (400 $\left.\mathrm{MHz}, \mathrm{CDCl}_{3}, 296 \mathrm{~K}\right) \delta(\mathrm{ppm})$ : $7.36(1 \mathrm{H}, \mathrm{d}, J=2.5 \mathrm{~Hz}, \mathrm{Ar}-\mathrm{H}), 7.29(1 \mathrm{H}, \mathrm{dd}, J=8.5,2.5 \mathrm{~Hz}, \mathrm{Ar}-\mathbf{H})$, 7.26 (1H, overlap, Ar-H), $7.23(1 \mathrm{H}, \mathrm{d}, J=2.5 \mathrm{~Hz}, \mathrm{Ar}-\mathbf{H}), 6.94(1 \mathrm{H}$, $\mathrm{d}, J=2.3 \mathrm{~Hz}, \operatorname{Ar}-\mathbf{H}), 6.93(1 \mathrm{H}, \mathrm{d}, J=2.3 \mathrm{~Hz}, \mathrm{Ar}-\mathbf{H}), 6.87(1 \mathrm{H}, \mathrm{d}, J=$ $2.3 \mathrm{~Hz}, \operatorname{Ar}-\mathbf{H}), 6.86(1 \mathrm{H}, \mathrm{d}, J=8.5 \mathrm{~Hz}, \operatorname{Ar}-\mathbf{H}), 6.61(1 \mathrm{H}, \mathrm{d}, J=$ $2.3 \mathrm{~Hz}, \mathrm{Ar}-\mathbf{H}), 3.96\left(3 \mathrm{H}, \mathrm{s},-\mathrm{OCH}_{3}\right), 3.94\left(3 \mathrm{H}, \mathrm{s},-\mathbf{O C H}_{3}\right), 3.93$ $\left(3 \mathrm{H}, \mathrm{s},-\mathrm{OCH}_{3}\right), 3.83\left(3 \mathrm{H}, \mathrm{s},-\mathrm{OCH}_{3}\right), 1.41(9 \mathrm{H}, \mathrm{s},-\mathrm{SO}-\boldsymbol{t}$-Bu), 1.25 (9H, s, t-Bu), 1.23 (9H, s, t-Bu), 1.07 (9H, s, t-Bu), 1.03 (9H, s, $\boldsymbol{t}$ Bu); ${ }^{13} \mathrm{C}$ NMR (100 MHz, $\left.\mathrm{CDCl}_{3}, 296 \mathrm{~K}\right) \delta$ (ppm): 158.2, 156.3, 154.6 , 153.5, 148.0, 147.9, 146.7, 144.3, 143.3, 131.9, 131.2, $130.8,129.9$, 128.9, 128.3, 127.6, 127.2, 127.1, 125.9, 125.7, 125.3, 125.0, 121.5, 110.7, 61.9, 60.9, 60.8, 56.1, 35.6, 34.7, 34.6, $34.6,34.3,31.5,31.5,31.2,31.2$, 31.1; IR (KBr) $\nu$ 2959, 2867, 1549, 1458, 1416, 1361, 1261, 1240, 1070, 1000, 908, 808, 731, $642 \mathrm{~cm}^{-1}$. HRMS $\left(\mathrm{ESI}^{+}\right):\left(\mathrm{C}_{48} \mathrm{H}_{66} \mathrm{O}_{4} \mathrm{~S}_{3}\right) \mathrm{m} / z$ calcd: $825.40154[\mathrm{M}+$ $\mathrm{Na}]^{+}, 841.37548[\mathrm{M}+\mathrm{K}]^{+}$, found: $825.40149[\mathrm{M}+\mathrm{Na}]^{+}, 841.37548$ $[\mathrm{M}+\mathrm{K}]^{+}$.

Compound 17. When the reaction of proximal disulfoxide $\mathbf{1 5}$ (30 mg, $37.1 \mathrm{mmol}$ ) with $n$-BuLi (20 eq., $2 \mathrm{M}$ in THF) was carried out under the similar conditions as described above in general procedure (quenched after $30 \mathrm{~min}$ with $3 \mathrm{~mL}$ of distilled water), trimer 17 was isolated from the reaction mixture as the main product (18 $\mathrm{mg}, 89 \%$, colourless oil) using preparative TLC with $\mathrm{CH}_{2} \mathrm{Cl}_{2}$ as an eluent. ${ }^{1} \mathrm{H} \mathrm{NMR}\left(400 \mathrm{MHz}, \mathrm{CDCl}_{3}, 296 \mathrm{~K}\right) \delta(\mathrm{ppm})$ : $7.28(2 \mathrm{H}, \mathrm{dd}, J=8.5,2.5 \mathrm{~Hz}, \mathrm{Ar}-\mathbf{H}), 7.24(2 \mathrm{H}, \mathrm{d}, J=2.5 \mathrm{~Hz}, \mathrm{Ar}-\mathbf{H})$, $6.86(4 \mathrm{H}, \mathrm{m}, \mathrm{Ar}-\mathrm{H}), 3.94\left(3 \mathrm{H}, \mathrm{s},-\mathrm{OCH}_{3}\right), 3.85\left(6 \mathrm{H}, \mathrm{s},-\mathrm{OCH}_{3}\right), 1.24$ (18H, s, $\boldsymbol{t}$-Bu), 1.05 (9H, s, $\boldsymbol{t}$-Bu); ${ }^{13} \mathrm{C}$ NMR (100 MHz, $\mathrm{CDCl}_{3}, 296$ K) $\delta(\mathrm{ppm}): 156.1,154.3,147.6,144.1,130.4,129.2,126.8,125.6$, 121.7, 110.5, 60.8, 56.0, 34.5, 34.2, 31.4, 31.1; IR (KBr) $\nu 2955$, 2915, 2868, 1730, 1489, 1459, 1260, 1245, 1070, 1024, $808 \mathrm{~cm}^{-1}$. HRMS $\left(\mathrm{ESI}^{+}\right):\left(\mathrm{C}_{33} \mathrm{H}_{44} \mathrm{O}_{3} \mathrm{~S}_{2}\right) \mathrm{m} / z$ calcd: $575.26241[\mathrm{M}+\mathrm{Na}]^{+}$, found: $575.26254[\mathrm{M}+\mathrm{Na}]^{+}$.

Compound 18. When the reaction of distal disulfoxide 16 (30 mg, $37.1 \mathrm{mmol}$ ) with $n$-BuLi (20 eq., $2 \mathrm{M}$ in THF) was carried out under the similar conditions as described above in general procedure (quenched after $30 \mathrm{~min}$ with $3 \mathrm{~mL}$ of distilled water), trimer 18 was isolated from the reaction mixture as the main product (24 mg, 90\%, colourless oil) using preparative TLC with 
$\mathrm{CH}_{2} \mathrm{Cl}_{2}$ as an eluent. ${ }^{1} \mathrm{H} \mathrm{NMR}\left(400 \mathrm{MHz}, \mathrm{CDCl}_{3}, 296 \mathrm{~K}\right) \delta(\mathrm{ppm})$ : $7.24(2 \mathrm{H}, \mathrm{dd}, J=8.5,2.5 \mathrm{~Hz}, \operatorname{Ar}-\mathbf{H}), 7.10(2 \mathrm{H}, \mathrm{d}, J=2.5 \mathrm{~Hz}, \mathrm{Ar}-\mathbf{H})$, $6.84(2 \mathrm{H}, \mathrm{d}, J=8.5 \mathrm{~Hz}, \mathrm{Ar}-\mathrm{H}), 3.85\left(6 \mathrm{H}, \mathrm{s},-\mathrm{OCH}_{3}\right), 1.20(18 \mathrm{H}, \mathrm{s}, \boldsymbol{t}$ Bu); ${ }^{13} \mathrm{C}$ NMR (100 MHz, $\left.\mathrm{CDCl}_{3}, 296 \mathrm{~K}\right) \delta(\mathrm{ppm}): 155.7,143.9$, 129.4, 124.9, 121.9, 110.2, 55.9, 34.1, 31.4; IR (KBr) v 2956, 2866, 1488, 1461, 1439, 1392, 1246, 1066, 1023, $806 \mathrm{~cm}^{-1}$. HRMS $\left(\mathrm{ESI}^{+}\right):\left(\mathrm{C}_{22} \mathrm{H}_{30} \mathrm{O}_{2} \mathrm{~S}\right) \mathrm{m} / z$ calcd: $359.20393[\mathrm{M}+\mathrm{H}]^{+}, 381.18587[\mathrm{M}$ $+\mathrm{Na}]^{+}, 397.15981[\mathrm{M}+\mathrm{K}]^{+}$, found: 359.20386 $[\mathrm{M}+\mathrm{H}]^{+}$, $381.18622[\mathrm{M}+\mathrm{Na}]^{+}, 397.15955[\mathrm{M}+\mathrm{K}]^{+}$.

\section{Deuteration experiments}

Compound 9. When the reaction mixture was quenched after $30 \mathrm{~s}$ with $\mathrm{D}_{2} \mathrm{O}$, mono-deuterated derivative 9 was isolated as the main product (71\%, colourless oil). ${ }^{1} \mathrm{H}$ NMR $(400 \mathrm{MHz}$, $\left.\mathrm{CDCl}_{3}, 296 \mathrm{~K}\right) \delta$ (ppm): $7.30(2 \mathrm{H}, \mathrm{m}, \mathrm{Ar}-\mathrm{H}), 7.26$ (2H, overlap, ArH), $6.98(2 \mathrm{H}, \mathrm{d}, J=2.4 \mathrm{~Hz}, \operatorname{Ar}-\mathbf{H}), 6.88(2 \mathrm{H}, \mathrm{m}, \operatorname{Ar}-\mathbf{H}), 6.80(1 \mathrm{H}$, $\mathrm{m}, \mathrm{Ar}-\mathrm{H}), 3.93\left(6 \mathrm{H}, \mathrm{s},-\mathrm{OCH}_{3}\right), 3.83\left(6 \mathrm{H}, \mathrm{s},-\mathrm{OCH}_{3}\right), 1.24(18 \mathrm{H}, \mathrm{s}$, $\boldsymbol{t}$-Bu), 1.07 (18H, s, $\boldsymbol{t}$-Bu); ${ }^{13} \mathrm{C}$ NMR (100 $\left.\mathrm{MHz} \mathrm{CDCl}_{3}, 296 \mathrm{~K}\right)$ $\delta$ (ppm): 156.32, 156.30, 154.67, 147.89, 144.27, 144.26, 130.88 $(2 \times), 129.95,129.94,128.85,127.81(2 \times), 127.25(2 \times), 125.95$, 125.86, 121.48, 121.44, 110.72, $61.00(2 \times), 56.09(2 \times), 34.64$ $(2 \times), 34.31(2 \times), 31.52(2 \times), 31.23(2 \times)$; IR (KBr) $\nu 2952,2929$, 2863, 1592, 1458, 1416, 1387, 1260, 1238, 1070, 996, 871, $807 \mathrm{~cm}^{-1}$. HRMS $\left(\mathrm{ESI}^{+}\right):\left(\mathrm{C}_{44} \mathrm{H}_{57} \mathrm{DO}_{4} \mathrm{~S}_{3}\right) \mathrm{m} / z$ calcd: $770.34522[\mathrm{M}$ $+\mathrm{Na}]^{+}, 786.31916[\mathrm{M}+\mathrm{K}]^{+}$, found: 770.34546 $[\mathrm{M}+\mathrm{Na}]^{+}$, $786.31763[\mathrm{M}+\mathrm{K}]^{+}$.

Compounds 10, 11, 13 and 14. The presence of the title compounds in the reaction mixtures were confirmed by HRMS $\left(\mathrm{ESI}^{+}\right)$analysis.

Compound 10. HRMS (ESI $\left.{ }^{+}\right):\left(\mathrm{C}_{48} \mathrm{H}_{64} \mathrm{D}_{2} \mathrm{O}_{5} \mathrm{~S}_{4}\right) \mathrm{m} / \mathrm{z}$ calcd: 853.39914 $[\mathrm{M}+\mathrm{H}]^{+}, 875.38108[\mathrm{M}+\mathrm{Na}]^{+}$; found: $853.39962[\mathrm{M}+$ $\mathrm{H}]^{+}, 875.38134[\mathrm{M}+\mathrm{Na}]^{+}$.

Compound 11. HRMS (ESI $\left.{ }^{+}\right): \mathrm{C}_{44} \mathrm{H}_{56} \mathrm{D}_{2} \mathrm{O}_{4} \mathrm{~S}_{3}, m / z$ calcd: $771.35150[\mathrm{M}+\mathrm{Na}]^{+}$; found: $771.34955[\mathrm{M}+\mathrm{Na}]^{+}$.

Compound 13. HRMS (ESI $\left.{ }^{+}\right): \mathrm{C}_{8} \mathrm{H}_{17} \mathrm{D}_{1} \mathrm{O}_{1} \mathrm{~S}_{1}, m / z$ calcd: 164.12139 $[\mathrm{M}+\mathrm{H}]^{+}$, found: $164.12170[\mathrm{M}+\mathrm{H}]^{+}$.

Compound 14. HRMS (ESI $\left.{ }^{+}\right): \mathrm{C}_{8} \mathrm{H}_{16} \mathrm{D}_{2} \mathrm{O}_{1} \mathrm{~S}_{1}, m / z$ calcd: 165.12767 $[\mathrm{M}+\mathrm{H}]^{+}$, found: $165.12774[\mathrm{M}+\mathrm{H}]^{+}$.

\section{General procedure for cleavage/substitution of thiacalixarene} monosulfoxide

Thiacalixarene 5 (50 mg, $0.063 \mathrm{mmol}$ ) was added to a $25 \mathrm{~mL}$ Schlenk flask and put under high vacuum (oil pump). After 20 minutes, the flask was filled several times with argon and anhydrous THF ( $5 \mathrm{~mL})$ was added through septum. The solution was cooled down to $-78{ }^{\circ} \mathrm{C}$ and $n$-BuLi (10 eq., $2 \mathrm{M}$ in THF) was added in one portion to the flask. The reaction mixture was stirred for 30 seconds at $-78{ }^{\circ} \mathrm{C}$, quenched by the addition of the corresponding electrophile, and stirred for $10 \mathrm{~min}$ at $-78{ }^{\circ} \mathrm{C}$. The cooling bath was then removed and the content of the flask was allowed to warm to RT. After the addition of distilled water $(3 \mathrm{~mL})$, the mixture was extracted with $\mathrm{CH}_{2} \mathrm{Cl}_{2}$ (3 $\times 15 \mathrm{~mL}$ ) and brine. Collected organic layers were washed with water $(50 \mathrm{~mL})$ and dried over $\mathrm{MgSO}_{4}$. The solvent was removed under reduced pressure to yield crude product which was further purified using preparative thin layer chromatography.
Compounds 19 and 20. The reaction mixture was quenched by the addition of dry benzaldehyde (4 eq., $0.026 \mathrm{~mL}, 0.252$ mmol). Compounds 19 (26 mg, 48\%, colourless oil) and 20 (17 mg, 28\%, colourless oil) were isolated using preparative TLC with $\mathrm{CH}_{2} \mathrm{Cl}_{2}$ as an eluent.

Data for 19. ${ }^{1} \mathrm{H}$ NMR (400 MHz, $\left.\mathrm{CDCl}_{3}, 296 \mathrm{~K}\right) \delta(\mathrm{ppm}): 7.41$ $(1 \mathrm{H}, \mathrm{d}, J=2.3 \mathrm{~Hz}, \mathrm{Ar}-\mathrm{H}), 7.39(2 \mathrm{H}, \mathrm{m}, \mathrm{Ar}-\mathbf{H}), 7.33(2 \mathrm{H}, \mathrm{t}, J=7.3 \mathrm{~Hz}$, Ar-H), 7.30 (1H, m, Ar-H), $7.26(2 \mathrm{H}, \mathrm{m}, \mathrm{Ar}-\mathbf{H}), 7.22(1 \mathrm{H}, \mathrm{d}, J=$ $2.3 \mathrm{~Hz}, \operatorname{Ar}-\mathbf{H}), 6.96(1 \mathrm{H}, \mathrm{d}, J=2.3 \mathrm{~Hz}, \mathrm{Ar}-\mathbf{H}), 6.95(1 \mathrm{H}, \mathrm{d}, J=2.3 \mathrm{~Hz}$, Ar-H), 6.87 (2H, m, Ar-H), 6.74 (1H, d, $J=2.3 \mathrm{~Hz}, \operatorname{Ar}-\mathbf{H}), 6.03$ $(1 \mathrm{H}, \mathrm{s}, \mathrm{CH}-\mathrm{OH}), 3.92\left(3 \mathrm{H}, \mathrm{s},-\mathrm{OCH}_{3}\right), 3.91\left(3 \mathrm{H}, \mathrm{s},-\mathrm{OCH}_{3}\right), 3.83$ $\left(3 \mathrm{H}, \mathrm{s},-\mathrm{OCH}_{3}\right), 3.57\left(3 \mathrm{H}, \mathrm{s},-\mathrm{OCH}_{3}\right), 2.94$ (1H, br.s, OH), 1.24 (9H, s, $\boldsymbol{t}$-Bu), 1.23 (9H, s, $\boldsymbol{t}$-Bu), 1.06 (9H, s, $\boldsymbol{t}$-Bu), 1.04 (9H, s, $\boldsymbol{t}$-Bu); ${ }^{13} \mathrm{C}$ NMR (100 MHz, $\left.\mathrm{CDCl}_{3}, 296 \mathrm{~K}\right) \delta$ (ppm): 156.25, 155.14, $154.58,153.94,148.00,147.93,147.80,144.16,143.88,137.20$, $131.06,130.84,129.99,128.97,128.38,128.33$, 127.77, 127.46, 127.42 , 127.18, 126.60, 126.42, 126.38, 125.91, 125.32, 121.21, 110.61, 72.95, 61.33, 60.85, 60.77, 55.97, 34.60, $34.49(2 \times), 34.17$, 31.40, 31.32, 31.10, 31.08; IR (KBr) $\nu$ 2960, 2868, 1725, 1549, 1459, 1417, 1362, 1262, 1163, 1070, 999, 734, $700 \mathrm{~cm}^{-1}$. HRMS $\left(\mathrm{ESI}^{+}\right)$: $\left(\mathrm{C}_{51} \mathrm{H}_{64} \mathrm{O}_{5} \mathrm{~S}_{3}\right) m / z$ calcd: $875.38081[\mathrm{M}+\mathrm{Na}]^{+}, 891.35475[\mathrm{M}+\mathrm{K}]^{+}$, found: $875.38116[\mathrm{M}+\mathrm{Na}]^{+}, 891.35321[\mathrm{M}+\mathrm{K}]^{+}$.

Data for 20. ${ }^{1} \mathrm{H}$ NMR (400 MHz, $\left.\mathrm{CDCl}_{3}, 296 \mathrm{~K}\right) \delta(\mathrm{ppm}): 7.42$ $(2 \mathrm{H}, \mathrm{d}, J=2.2 \mathrm{~Hz}, \operatorname{Ar}-\mathbf{H}), 7.38(4 \mathrm{H}, \mathrm{m}, \operatorname{Ar}-\mathbf{H}), 7.33(4 \mathrm{H}, \mathrm{t}, J=$ $7.5 \mathrm{~Hz}, \operatorname{Ar}-\mathbf{H}), 7.25(2 \mathrm{H}, \mathrm{t}, J=7.5 \mathrm{~Hz}, \operatorname{Ar}-\mathbf{H}), 7.23(2 \mathrm{H}, \mathrm{d}, J=$ $2.2 \mathrm{~Hz}, \operatorname{Ar}-\mathbf{H}), 6.92(2 \mathrm{H}, \mathrm{d}, J=2.2 \mathrm{~Hz}, \operatorname{Ar}-\mathbf{H}), 6.74(2 \mathrm{H}, \mathrm{d}, J=$ $2.2 \mathrm{~Hz}, \mathrm{Ar}-\mathbf{H}), 6.03(2 \mathrm{H}, \mathrm{s}, \mathrm{CH}-\mathrm{OH}), 3.91\left(6 \mathrm{H}, \mathrm{s},-\mathrm{OCH}_{3}\right), 3.57$ $\left(6 \mathrm{H}, \mathrm{s},-\mathrm{OCH}_{3}\right), 2.92$ (2H, br.s, $\left.-\mathrm{OH}\right), 1.24(18 \mathrm{H}, \mathrm{s}, \boldsymbol{t}-\mathbf{B u}), 1.03$ $\left(18 \mathrm{H}, \mathrm{s}, \boldsymbol{t}\right.$-Bu); ${ }^{13} \mathrm{C}$ NMR (100 $\left.\mathrm{MHz}, \mathrm{CDCl}_{3}, 296 \mathrm{~K}\right) \delta(\mathrm{ppm})$ : 155.19 , 153.99, 148.04, 147.95, 143.86, 137.22, 131.14, 130.32, 128.58, 128.38, 127.55, 127.42, 126.48, 126.44, 126.41, 125.38, 72.95, 61.34, 60.78, 34.60, 34.48, 31.32, 31.05; IR (KBr) $\nu 2961$, 2931, 2868, 1681, 1549, 1459, 1418, 1393, 1362, 1264, 1239, 1163, 1090, 999, 735, $699 \mathrm{~cm}^{-1}$. HRMS $\left(\mathrm{ESI}^{+}\right):\left(\mathrm{C}_{58} \mathrm{H}_{70} \mathrm{O}_{6} \mathrm{~S}_{3}\right) \mathrm{m} / \mathrm{z}$ calcd: $981.42267[\mathrm{M}+\mathrm{Na}]^{+}$, $997.39661[\mathrm{M}+\mathrm{K}]^{+}$, found: $981.42185[\mathrm{M}+\mathrm{Na}]^{+}, 997.39548[\mathrm{M}+\mathrm{K}]^{+}$.

Compounds 21 and 22. The reaction mixture was quenched by the addition of benzophenone (4 eq., $0.252 \mathrm{mmol}$ ). Compounds 21 (25 mg, 43\%, colourless oil) and 22 (17 mg, 24\%, colourless oil) were isolated using preparative TLC with $\mathrm{CH}_{2} \mathrm{Cl}_{2}$ as an eluent.

Data for 21. ${ }^{1} \mathrm{H}$ NMR (400 MHz, $\left.\mathrm{CDCl}_{3}, 296 \mathrm{~K}\right) \delta(\mathrm{ppm}): 7.41$ $(1 \mathrm{H}, \mathrm{d}, J=2.3 \mathrm{~Hz}, \mathrm{Ar}-\mathrm{H}), 7.39(2 \mathrm{H}, \mathrm{m}, \operatorname{Ar}-\mathbf{H}), 7.33(2 \mathrm{H}, \mathrm{t}, J=$ $7.3 \mathrm{~Hz}, \mathrm{Ar}-\mathbf{H}), 7.30$ (1H, m, Ar-H), $7.26(2 \mathrm{H}, \mathrm{m}, \mathrm{Ar}-\mathbf{H}), 7.22(1 \mathrm{H}, \mathrm{d}$, $J=2.3 \mathrm{~Hz}, \operatorname{Ar}-\mathbf{H}), 6.96(1 \mathrm{H}, \mathrm{d}, J=2.3 \mathrm{~Hz}, \operatorname{Ar}-\mathbf{H}), 6.95(1 \mathrm{H}, \mathrm{d}, J=$ $2.3 \mathrm{~Hz}, \operatorname{Ar}-\mathbf{H}), 6.87$ (2H, m, Ar-H), $6.74(1 \mathrm{H}, \mathrm{d}, J=2.3 \mathrm{~Hz}, \mathrm{Ar}-\mathbf{H})$, $6.03(1 \mathrm{H}, \mathrm{s}, \mathrm{CH}-\mathrm{OH}), 3.92\left(3 \mathrm{H}, \mathrm{s},-\mathrm{OCH}_{3}\right), 3.91\left(3 \mathrm{H}, \mathrm{s},-\mathrm{OCH}_{3}\right)$, $3.83\left(3 \mathrm{H}, \mathrm{s},-\mathrm{OCH}_{3}\right), 3.57\left(3 \mathrm{H}, \mathrm{s},-\mathrm{OCH}_{3}\right), 2.94$ (1H, br.s, OH), 1.24 (9H, s, t-Bu), 1.23 (9H, s, $\boldsymbol{t}$-Bu), 1.06 (9H, s, $\boldsymbol{t}$-Bu), 1.04 (9H, s, $\boldsymbol{t}$-Bu); ${ }^{13} \mathrm{C} \mathrm{NMR}\left(100 \mathrm{MHz}, \mathrm{CDCl}_{3}, 296 \mathrm{~K}\right) \delta(\mathrm{ppm}): 156.32,156.25$, 154.54, 153.41, 148.02, 147.79, 146.79, 146.47, 144.16, 141.84, 132.08, 130.85, 130.75, 129.99, 128.77, 128.52, 128.34, 127.93, $127.85,127.69,127.36,127.16,126.55,126.00,125.91,125.32$, 121.20, 110.61, 82.43, 60.85 (2×), 60.71, 55.97, 34.54, 34.49,

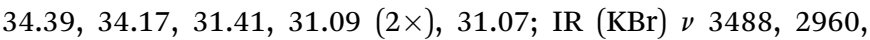
2867, 1595, 1549, 1459, 1393, 1362, 1262, 1245, 1070, 1023, 997, 
908, 733, $699 \mathrm{~cm}^{-1}$. HRMS $\left(\mathrm{ESI}^{+}\right):\left(\mathrm{C}_{57} \mathrm{H}_{68} \mathrm{O}_{5} \mathrm{~S}_{3}\right) \mathrm{m} / z$ calcd: 951.41211 $[\mathrm{M}+\mathrm{Na}]^{+}, 967.38605[\mathrm{M}+\mathrm{K}]^{+}$, found: $951.41287[\mathrm{M}+$ $\mathrm{Na}]^{+}, 967.38480[\mathrm{M}+\mathrm{K}]^{+}$.

Data for 22. ${ }^{1} \mathrm{H}$ NMR (400 MHz, $\left.\mathrm{CDCl}_{3}, 296 \mathrm{~K}\right) \delta(\mathrm{ppm}): 7.32$ (16H, m, Ar-H), 7.29 (4H, m, Ar-H), $7.28(2 \mathrm{H}, \mathrm{d}, J=2.5 \mathrm{~Hz}, \mathrm{Ar}-\mathbf{H})$, $6.89(2 \mathrm{H}, \mathrm{d}, J=2.3 \mathrm{~Hz}, \mathrm{Ar}-\mathrm{H}), 6.66(2 \mathrm{H}, \mathrm{d}, J=2.3 \mathrm{~Hz}, \mathrm{Ar}-\mathbf{H}), 6.59$ $(2 \mathrm{H}, \mathrm{d}, J=2.5 \mathrm{~Hz}, \mathrm{Ar}-\mathrm{H}), 5.62(2 \mathrm{H}, \mathrm{s},-\mathrm{OH}), 3.92\left(6 \mathrm{H}, \mathrm{s},-\mathrm{OCH}_{3}\right)$, $3.15\left(6 \mathrm{H}, \mathrm{s},-\mathrm{OCH}_{3}\right), 1.08(18 \mathrm{H}, \mathrm{s}, \boldsymbol{t}$-Bu $), 1.07(18 \mathrm{H}, \mathrm{s}, \boldsymbol{t}-\mathrm{Bu}) ;{ }^{13} \mathrm{C}$ NMR (100 MHz, $\left.\mathrm{CDCl}_{3}, 296 \mathrm{~K}\right) \delta$ (ppm): 156.38, 153.41, 148.05, 146.81, 146.44, 141.88, 132.16, 130.90, 128.38, 128.27, 127.92, $127.85,127.37,127.16,125.86,125.35,82.43,60.86,60.74$, 34.52, 34.39, 31.06, 31.04; IR (KBr) $\nu$ 3493, 2961, 2867, 1549, 1460, 1419, 1393, 1362, 1265, 1232, 1159, 1091, 1032, 996, 908, 733, $700 \mathrm{~cm}^{-1}$. HRMS (ESI $\left.{ }^{+}\right):\left(\mathrm{C}_{70} \mathrm{H}_{78} \mathrm{O}_{6} \mathrm{~S}_{3}\right) \mathrm{m} / z$ calcd: 1133.48527 $[\mathrm{M}+\mathrm{Na}]^{+}, 1149.45921[\mathrm{M}+\mathrm{K}]^{+}$, found: 1133.48591 $[\mathrm{M}+\mathrm{Na}]^{+}, 1149.45857[\mathrm{M}+\mathrm{K}]^{+}$.

Compounds 23 and 24. The reaction mixture was quenched by the addition of ethyl chloroformate (4 eq., $0.024 \mathrm{~mL}, 0.252$ mmol). Compounds 23 (22 mg, 42\%, colourless oil) and 24 (11 mg, 19\%, colourless oil) were isolated using preparative TLC with $\mathrm{CH}_{2} \mathrm{Cl}_{2}$ as an eluent.

Data for 23. ${ }^{1} \mathrm{H}$ NMR (400 MHz, $\left.\mathrm{CDCl}_{3}, 296 \mathrm{~K}\right) \delta(\mathrm{ppm}): 7.67$ $(1 \mathrm{H}, \mathrm{d}, J=2.4 \mathrm{~Hz}, \mathrm{Ar}-\mathbf{H}), 7.30(1 \mathrm{H}, \mathrm{dd}, J=8.5,2.5 \mathrm{~Hz}, \mathrm{Ar}-\mathbf{H}), 7.28$ $(1 \mathrm{H}, \mathrm{d}, J=2.4 \mathrm{~Hz}, \mathrm{Ar}-\mathrm{H}), 7.24(1 \mathrm{H}, \mathrm{d}, J=2.5 \mathrm{~Hz}, \mathrm{Ar}-\mathrm{H}), 7.01(3 \mathrm{H}$, $\mathrm{m}, \operatorname{Ar}-\mathbf{H}), 6.89(1 \mathrm{H}, \mathrm{d}, J=2.4 \mathrm{~Hz}, \operatorname{Ar}-\mathbf{H}), 6.87(1 \mathrm{H}, \mathrm{d}, J=8.5 \mathrm{~Hz}$, $\mathrm{Ar}-\mathrm{H}), 4.40\left(2 \mathrm{H}, J=7.2 \mathrm{~Hz},-\mathrm{OCH}_{2} \mathrm{CH}_{3}\right), 3.92\left(6 \mathrm{H}, \mathrm{s},-\mathrm{OCH}_{3}\right)$, $3.90\left(3 \mathrm{H}, \mathrm{s},-\mathrm{OCH}_{3}\right), 3.83\left(3 \mathrm{H}, \mathrm{s},-\mathrm{OCH}_{3}\right), 1.41(3 \mathrm{H}, J=7.2 \mathrm{~Hz}$, $-\mathrm{OCH}_{2} \mathbf{C H}_{3}$ ), 1.24 (9H, s, $\boldsymbol{t}$-Bu), 1.21 (9H, s, $\boldsymbol{t}$-Bu), 1.10 (9H, s, $\boldsymbol{t}$ Bu), 1.08 (9H, s, t-Bu); ${ }^{13} \mathrm{C} \mathrm{NMR}\left(100 \mathrm{MHz}, \mathrm{CDCl}_{3}, 296 \mathrm{~K}\right)$ $\delta$ (ppm): 166.6, 156.3, 155.8, 155.2, 154.8, 148.2, 147.9, 147.3, 144.2 , 132.7, 130.9, 130.6, 130.2, 129.9, 128.7, 128.4, 128.3, 128.1, 128.0, 127.3, 127.0, 126.0, 125.2, 121.1, 110.6, 62.1, 61.3, 61.0, 60.9, 56.0, 34.6, 34.5, 34.4, 34.2, 31.4, $31.2(2 \times), 31.1,14.3$; IR $(\mathrm{KBr}) \nu$ 2960, 1727, 1549, 1460, 1418, 1393, 1364, 1259, 1092, 1000, 875, $791 \mathrm{~cm}^{-1}$. HRMS $\left(\mathrm{ESI}^{+}\right):\left(\mathrm{C}_{47} \mathrm{H}_{62} \mathrm{O}_{6} \mathrm{~S}_{3}\right) \mathrm{m} / z$ calcd: 841.36007 $[\mathrm{M}+\mathrm{Na}]^{+}, 857.33401[\mathrm{M}+\mathrm{K}]^{+}$, found: $841.36038[\mathrm{M}+$ $\mathrm{Na}]^{+}, 857.33298[\mathrm{M}+\mathrm{K}]^{+}$.

Data for 24. ${ }^{1} \mathrm{H}$ NMR (400 MHz, $\left.\mathrm{CDCl}_{3}, 296 \mathrm{~K}\right) \delta(\mathrm{ppm}): 7.68$ $(2 \mathrm{H}, \mathrm{d}, J=2.5 \mathrm{~Hz}, \mathrm{Ar}-\mathbf{H}), 7.25(2 \mathrm{H}, \mathrm{d}, J=2.5 \mathrm{~Hz}, \operatorname{Ar}-\mathbf{H}), 7.06(2 \mathrm{H}$, $\mathrm{d}, J=2.4 \mathrm{~Hz}, \mathrm{Ar}-\mathrm{H}), 7.02(2 \mathrm{H}, \mathrm{d}, J=2.4 \mathrm{~Hz}, \mathrm{Ar}-\mathbf{H}), 4.40(2 \mathrm{H}, J=$ $\left.7.2 \mathrm{~Hz},-\mathrm{OCH}_{2} \mathrm{CH}_{3}\right), 3.92\left(6 \mathrm{H}, \mathrm{s},-\mathrm{OCH}_{3}\right), 3.89\left(6 \mathrm{H}, \mathrm{s},-\mathrm{OCH}_{3}\right)$, $1.41\left(6 \mathrm{H}, J=7.2 \mathrm{~Hz},-\mathrm{OCH}_{2} \mathbf{C H}_{3}\right), 1.22(18 \mathrm{H}, \mathrm{s}, \boldsymbol{t}$-Bu), 1.11 $(18 \mathrm{H}, \mathrm{s}, \boldsymbol{t}$-Bu $) ;{ }^{13} \mathrm{C} \mathrm{NMR}\left(100 \mathrm{MHz}, \mathrm{CDCl}_{3}, 296 \mathrm{~K}\right) \delta(\mathrm{ppm}): 166.5$, $155.9,155.4,148.3,147.3,133.2$, 133.0, 132.9, 130.4, 129.1, 128.9, 128.8, 128.7, 128.6, 127.4, 127.1, 125.3, 62.2, 61.3, 61.0, 34.6, 34.5, $31.2(2 \times)$, 14.3; IR (KBr) $\nu$ 2962, 1728, 1550, 1464, 1419, 1392, 1366, 1261, 1093, 1003, 876, $791 \mathrm{~cm}^{-1}$. HRMS (ESI $\left.{ }^{+}\right)$: $\left(\mathrm{C}_{50} \mathrm{H}_{66} \mathrm{O}_{8} \mathrm{~S}_{3}\right) \mathrm{m} / z$ calcd: $913.38120[\mathrm{M}+\mathrm{Na}]^{+}, 929.35514[\mathrm{M}+\mathrm{K}]^{+}$, found: $913.38123[\mathrm{M}+\mathrm{Na}]^{+}, 929.35513[\mathrm{M}+\mathrm{K}]^{+}$.

Compounds 25 and 26. The reaction mixture was quenched by the introducing (via cannula) of gaseous $\mathrm{CO}_{2}$ directly sublimed from dry ice and dried over $\mathrm{CaCl}_{2}$. Compound 25 (22 mg, 44\%, colourless oil) was isolated using preparative TLC with $\mathrm{CH}_{2} \mathrm{Cl}_{2}$-methanol (50:1, v/v) mixture as an eluent. The presence of derivative $\mathbf{2 6}$ in the crude reaction mixture was confirmed by MS, nevertheless, it has never been isolated in a pure form.
Data for 25. ${ }^{1} \mathrm{H}$ NMR (400 MHz, $\left.\mathrm{CDCl}_{3}, 296 \mathrm{~K}\right) \delta$ (ppm): 10.79 $(1 \mathrm{H}$, br s, $-\mathrm{COOH}), 8.07(1 \mathrm{H}, \mathrm{d}, J=2.4 \mathrm{~Hz}, \mathrm{Ar}-\mathrm{H}), 7.41(1 \mathrm{H}, \mathrm{d}, J=$ $2.4 \mathrm{~Hz}, \mathrm{Ar}-\mathrm{H}), 7.31$ (1H, dd, $J=8.5,2.4 \mathrm{~Hz}, \mathrm{Ar}-\mathbf{H}), 7.29(1 \mathrm{H}, \mathrm{d}, J=$ $2.4 \mathrm{~Hz}, \mathrm{Ar}-\mathrm{H}), 7.02(1 \mathrm{H}, \mathrm{d}, J=2.4 \mathrm{~Hz}, \mathrm{Ar}-\mathrm{H}), 7.00(1 \mathrm{H}, \mathrm{d}, J=2.4 \mathrm{~Hz}$, Ar-H), $6.91(1 \mathrm{H}, \mathrm{d}, J=2.4 \mathrm{~Hz}, \mathrm{Ar}-\mathbf{H}), 6.89(1 \mathrm{H}, \mathrm{d}, J=2.4 \mathrm{~Hz}, \mathrm{Ar}-\mathrm{H})$, $6.88(1 \mathrm{H}, \mathrm{d}, J=8.5 \mathrm{~Hz}, \operatorname{Ar}-\mathbf{H}), 4.09\left(3 \mathrm{H}, \mathrm{s},-\mathrm{OCH}_{3}\right), 3.92(3 \mathrm{H}, \mathrm{s}$, $\left.-\mathrm{OCH}_{3}\right), 3.91\left(3 \mathrm{H}, \mathrm{s},-\mathrm{OCH}_{3}\right), 3.83\left(3 \mathrm{H}, \mathrm{s},-\mathrm{OCH}_{3}\right), 1.24(18 \mathrm{H}, \mathrm{s}, \mathrm{t}$ Bu), 1.09 (9H, s, $t$-Bu), 1.08 (9H, s, $t$-Bu); ${ }^{13} \mathrm{C}$ NMR (100 MHz, $\left.\mathrm{CDCl}_{3}, 296 \mathrm{~K}\right) \delta(\mathrm{ppm}):$ 165.2, 156.4, 155.5, 154.8, 154.6, 149.2, 148.4, $147.9(2 \times), 144.2$, 135.7, 131.1, 130.5, 130.4, 129.2, 128.9, $128.3(2 \times), 127.6,127.5,127.4,126.1,122.0,120.9,110.7,63.0$, $61.0,60.9,55.9,34.8,34.6,34.5,34.2,31.4,31.2$, 31.1, 31.0; IR $(\mathrm{KBr}) \nu$ 2960, 2868, 1692, 1549, 1459, 1417, 1392, 1362, 1260, 1070, 999, 907, 808, $730 \mathrm{~cm}^{-1}$. HRMS (ESI $\left.{ }^{+}\right):\left(\mathrm{C}_{45} \mathrm{H}_{58} \mathrm{O}_{6} \mathrm{~S}_{3}\right) \mathrm{m} / z$ calcd: 813.32877 [M + Na $]^{+}$, found: $813.32880[\mathrm{M}+\mathrm{Na}]^{+}$.

Data for 26. HRMS $\left(\mathrm{ESI}^{+}\right):\left(\mathrm{C}_{46} \mathrm{H}_{58} \mathrm{O}_{8} \mathrm{~S}_{3}\right) \mathrm{m} / z$ calcd: 857.31860 $[\mathrm{M}+\mathrm{Na}]^{+}$, found: $857.31805[\mathrm{M}+\mathrm{Na}]^{+}$.

\section{$\mathrm{X}$-ray measurement}

Crystallographic data for $\mathbf{6}$. The structure of compound 6 was measured using D8 VENTURE equipped with Photon CMOS detector with $\mathrm{Cu}-\mathrm{K} \alpha(\lambda=1.54178 \AA)$ radiation at $180 \mathrm{~K}$. The structure was in monoclinic system, $C c$ space group with lattice parameters $a=12.3117$ (7) $\mathrm{\AA}, b=20.2840$ (7) $\mathrm{A}, c=18.3956$ (8) $\mathrm{\AA}$, $\beta=109.3246(18)^{\circ}, Z=4, V=4335.1(4) \AA^{3}, D_{\mathrm{c}}=1.145 \mathrm{~g} \mathrm{~cm}^{-3}$, $\mu(\mathrm{Cu}-\mathrm{K} \alpha)=1.86 \mathrm{~mm}^{-1}$. The data reduction and absorption correction were done with Apex3 software. ${ }^{17}$ The structure was solved by charge flipping methods ${ }^{\mathbf{1 8}}$ and refined by full matrix least squares on $F$ squared value using Crystals software ${ }^{\mathbf{1 9}}$ to final values $R=0.026$ an $\mathrm{w} R=0.070$ using 7775 independent reflections $\left(\Theta_{\max }=68.5^{\circ}\right), 587$ parameters and 204 restraint. MCE software ${ }^{20}$ was used for visualization of residual electron density maps. According to common practice the hydrogen atoms attached to carbon atoms were place geometrically with $U_{\text {iso }}(\mathrm{H})$ in range 1.2-1.5 $U_{\text {eq }}$ of parent atom (C). The absolute structure was confirmed by Flack parameter ${ }^{21}$ calculation with resulting value 0.064(8). The disordered functional groups were refined with restrained geometry and occupancy constrained to full for each atomic position. The structure was deposited into Cambridge Structural Database under number CCDC 1554286. $\dagger$

\section{Conflicts of interest}

There are no conflicts to declare.

\section{Acknowledgements}

This research was supported by the Czech Science Foundation (Grant 17-18108S). Financial support from specific university research (MSMT 20/2017) is also acknowledged.

\section{Notes and references}

1 H. Kumagai, M. Hasegawa, S. Miyanari, Y. Sugawa, Y. Sato, T. Hori, S. Ueda, H. Kamiyama and S. Miyano, Tetrahedron Lett., 1997, 38, 3971. 
2 For selected books on calixarenes and their applications, see: (a) Calixarenes and Beyond, ed. P. Neri, J. L. Sessler and M. X. Wang, Springer Int. Publishing, Switzerland, 2016; (b) C. D. Gutsche, Calixarenes An introduction, The Royal Society of Chemistry, Thomas Graham House, Cambridge, 2nd edn, 2008; (c) Calixarenes in the Nanoworld, ed. J. Vicens, J. Harrowfield and L. Backlouti, Springer, Dordrecht, 2007; (d) Calixarenes 2001, ed. Z. Asfari, V. Böhmer, J. Harrowfield and J. Vicens, Kluwer Academic Publishers, Dordrecht, 2001; (e) L. Mandolini and R. Ungaro, Calixarenes in Action, Imperial College Press, London, 2000.

3 For selected reviews on thiacalixarenes, see: $(a)$ M. Zhao, J. Lv and D.-S. Guo, RSC Adv., 2017, 7, 10021; (b) R. Kumar, Y. O. Lee, V. Bhalla, M. Kumar and J. S. Kim, Chem. Soc. Rev., 2014, 43, 4824; (c) N. Morohashi, F. Narumi, N. Iki, T. Hattori and S. Miyano, Chem. Rev., 2006, 106, 5291; (d) P. Lhotak, Eur. J. Org. Chem., 2004, 1675.

4 (a) O. Kundrat, I. Cisarova, S. Böhm, M. Pojarova and P. Lhotak, J. Org. Chem., 2009, 74, 4592; (b) O. Kundrat, H. Dvorakova, I. Cisarova, M. Pojarova and P. Lhotak, Org. Lett., 2009, 11, 4188; (c) O. Kundrat, H. Dvorakova, V. Eigner and P. Lhotak, J. Org. Chem., 2010, 75, 407.

5 (a) O. Kundrat, J. Kroupa, S. Böhm, J. Budka, V. Eigner and P. Lhotak, J. Org. Chem., 2010, 75, 8372; (b) O. Kundrat, H. Dvorakova, S. Böhm, V. Eigner and P. Lhotak, J. Org. Chem., 2012, 77, 2272.

6 (a) O. Kundrat, V. Eigner, H. Dvorakova and P. Lhotak, Org. Lett., 2011, 13, 4032; (b) O. Kundrat, H. Dvorakova, S. Böhm, V. Eigner and P. Lhotak, J. Org. Chem., 2012, 77, 2272 .

7 (a) H. Katagiri, T. Hattori, N. Morohashi, N. Iki and S. Miyano, J. Org. Chem., 2007, 72, 8327; (b) G. Mislin, E. Graf, M. W. Hosseini, A. De Cian and J. Fischer, Tetrahedron Lett., 1999, 40, 1129; (c) H. Katagiri, N. Iki, T. Hattori, C. Kabuto and S. Miyano, J. Am. Chem. Soc., 2001, 123, 779; (d) N. Morohashi, H. Katagiri, N. Iki, Y. Yamane, C. Kabuto, T. Hattori and S. Miyano, J. Org. Chem., 2003, 68, 2324; (e) N. Morohashi, N. Iki, C. Kabuto and S. Miyano, Tetrahedron Lett., 2000, 41, 2933; (f) N. Morohashi, N. Iki, T. Onodera, C. Kabuto and S. Miyano, Tetrahedron Lett., 2000, 41, 5093; $(g)$ P. Lhotak, Tetrahedron, 2001, 57, 4775.

$8 \mathrm{~J}$. Miksatko, V. Eigner, H. Dvorakova and P. Lhotak, Tetrahedron Lett., 2016, 57, 3781.

9 J. Miksatko, V. Eigner, M. Kohout and P. Lhotak, Tetrahedron Lett., 2017, 58, 1687.

10 J. Clayden, Directed Metallation of Aromatic Compounds, in Patai's Chemistry of Functional Groups, John Wiley \& Sons, Ltd, 2009.

11 L. Melzig, C. B. Rauhut, N. Naredi-Rainer and P. Knochel, Chem.-Eur. J., 2011, 17, 5362.

12 (a) N. Furukawa, S. Ogawa, K. Matsumura and H. Fujihara, J. Org. Chem., 1991, 56, 6341; (b) S. Oae, T. Kawai and N. Furukawa, Phosphorus Sulfur Relat. Elem., 1987, 34, 123; (c) T. Shibutani, H. Fujihara and N. Furukawa, Heteroat. Chem., 1991, 2, 521.

13 N. Furukawa and S. Sato, Top. Curr. Chem., 1999, 205, 90.

14 J. Clayden, C. C. Stimson and M. Keenan, Chem. Commun., 2006, 1393.

15 For the formation of dianions from dialkyl sulfoxides, see e.g.: (a) E. M. Kaiser, R. D. Beard and C. R. Hauser, J. Organomet. Chem., 1973, 59, 53; (b) C. M. Thompson, Dianion Chemistry in Organic Synthesis, CRC Press, Boca Raton, 1994.

16 (a) T. Sone, K. Moriya and Y. Ohba, Bull. Chem. Soc. Jpn., 1991, 64, 576; (b) T. Sone, Y. Ohba, K. Moriya, H. Kumada and K. Ito, Tetrahedron, 1997, 53, 10689.

17 Bruker, APEX3, SAINT, Bruker AXS Inc., Madison, Wisconsin, USA, 2015.

18 L. Palatinus and G. Chapuis, J. Appl. Crystallogr., 2007, 40, 786.

19 P. W. Betteridge, J. R. Carruthers, R. I. Cooper, K. Prout and D. J. Watkin, J. Appl. Crystallogr., 2003, 36, 1487.

20 J. Rohlicek and M. Husak, J. Appl. Crystallogr., 2007, 40, 600. 21 H. D. Flack, Acta Crystallogr., Sect. A: Found. Crystallogr., 1983, 39, 876. 